\title{
Specific and nonspecific transfer: Effects of nonreward, delay of reward, and punishment sequences on persistence
}

\author{
NABIL F. HADDAD \\ University of Montana, Missoula, Montana 59812 \\ ROGER L. MELLGREN \\ University of Oklahoma, Norman, Oklahoma 73019 \\ and \\ JOHN WALKENBACH \\ University of Montana, Missoula, Montana 59812
}

\begin{abstract}
Two experiments were conducted to test the effects of training with different aversive events on rats' subsequent persistence of responding. In Experiment 1, it was found that training with the same aversive event (nonreward) as that encountered during extinction (specific transfer) resulted in higher resistance to extinction than did training with different combinations of nonreward, punishment, and delay (nonspecific transfer). Experiment 2 indicated that these effects are controlled primarily by sequential variables and that persistence effects generated by the same or different aversive events as those encountered during extinction summate. A theoretical framework emphasizing the role of generalization decrement and sequential variables was invoked to account for these results.
\end{abstract}

Studies investigating the effects of intermittent punishment (e.g., Banks, 1966a, 1966b; Brown \& Wagner, 1964) and the effects of partial delay (e.g., Mellgren, Haddad, Williams, \& Conkright, 1975) indicate that the intermittent use of any of these events during the acquisition of a response results in increased persistence when that persistence is measured by continuous administration of the same event encountered during acquisition. On the other hand, when the aversive events encountered during the training phase and the testing phase are different, the resulting increase in persistence is often found to be marginal and sometimes nonexistent (e.g., Banks \& Torney, 1969; Brown \& Wagner, 1964; Dyck, Mellgren, \& Nation, 1974).

Most explanations used to account for these results have relied on drawing analogies between the counterconditioning of anticipatory frustration and anticipatory fear or pain (e.g., Amsel, 1972; D’Amato, 1969; Wagner, 1969). This class of theoretical explanation has not been totally successful in accounting for the results reported in studies investigating the transfer of persistence from training with one aversive event (e.g., punishment) to testing with a qualitatively different aversive event (e.g., nonreward). For example, frustration-type explanations do not account for the important role that

Requests for reprints should be sent to Nabil Haddad, Department of Psychology, University of Montana, Missoula, Montana 59812. sequential variables play in persistence. The overwhelming weight of recent evidence indicates that Capaldi's $(1967,1970)$ sequential theory possesses strong predictive powers not only in the case of the partial reinforcement effect, but also in the case of punishment (Capaldi \& Levy, 1972; Dyck et al., 1974) and in the case of delay of reinforcement (Mellgren et al., 1975).

The purpose of the present investigation was to construct an explanatory mechanism that would make use of the predictive powers of the sequential theory and then test specific predictions derived from the new approach. It is assumed here that an aversive event such as nonreward or punishment results in a memory of that event, and when the instrumental response is subsequently reinforced, a connection between the memory and the response is strengthened (habit strength is increased). The level of habit strength that may then generalize to the testing period depends on the similarity of aversive events encountered during the training phase and those encountered during the testing phase and on the absolute level of habit strength conditioned during the training phase. In addition, the theoretical approach proposed here consists of three basic assumptions: (1) Transfer of persistence effects are divided into two classes to be called specific and nonspecific transfer. Specific transfer occurs when the aversive events encountered in the training and the testing phases are the same; nonspecific transfer occurs when these aversive events are different. (2) Animals that experience 
specific transfer suffer less generalization decrement than do animals that experience nonspecific transfer (e.g., Brown \& Wagner, 1964; Dyck et al., 1974; Mellgren et al., 1975). (3) Habit strengths established through associations with different aversive events and undergoing specific and/or nonspecific transfer summate to increase the level of persistence.

\section{EXPERIMENT 1}

To provide a test of the assumptions outlined above, four groups of rats were trained to traverse a straightalley runway under different combinations of nonreward, punishment, and delay of reward ("aversive events") mixed with immediately rewarded, nonpunished trials and were then tested in an extinction phase. During the first phase, the three experimental groups received reinforcement schedules consisting of the same pattern and total number of transitions from aversive event-to-reward trials, and the control group received a CRF schedule. The three experimental groups differed only in the type of aversive event-to-reward trials transitions they received. One group received transitions from nonreward to reward (N-R) only, a second group received a combination of punishmentto-reward (P-R) and delayed reward-to-reward (D-R) transitions, and the third group received a combination of N-R, P-R, and D-R transitions. The assumptions outlined above predict that all three experimental groups would be more resistant to extinction than the CRF control group, due to specific transfer for the N-R group, nonspecific transfer for the P-R/D-R group, and the summation of both specific and nonspecific transfer for the N-R/P-R/D-R group. More important, the assumptions presented here predict different levels of resistance to extinction for the three experimental groups, despite the equal number of aversive events and the equal number of transitions these groups receive in the first, acquisition phase.

\section{Method}

Subjects. The subjects were 32 naive male albino rats, 80 days old, of the Sprague-Dawley strain, purchased from the Holtzman Company, Madison, Wisconsin. The animals were randomly assigned to one of four groups ( $n=8 /$ group).

Apparatus. The apparatus consisted of a straight-alley runway constructed of clear Plexiglas with a grid floor. It measured $136 \times 10 \times 13 \mathrm{~cm}$ and was divided into a $28-\mathrm{cm}$-long startbox, an $80-\mathrm{cm}$-long run section, and a $28-\mathrm{cm}$ tong goalbox. A food cup constructed of $1-\mathrm{cm}$-thick Plexiglas was mounted at the far end of the goalbox. Immediately preceding the food cup, a manually retractable guillotine door constructed of white translucent plastic served to delay access to food on delay-ofreward trials. Start, run, and goal times were measured to the nearest $.01 \mathrm{sec}$ by means of three Standard timers controlled by a microswitch, located at the translucent guillotine door of the start section, and a series of three photoelectric cells located $20 \mathrm{~cm}$ beyond the startbox door, $17 \mathrm{~cm}$ in front of the goalbox door, and $11 \mathrm{~cm}$ inside the goalbox. The sum of these three measures for each trial yielded total time, and the reciprocais of all four measures were then calculated to yield start, run, goal, and total speeds. On punished trials, a Model 700 Grason-
Stadler shock generator delivered $.5 \mathrm{sec}$ of $.2-\mathrm{mA}$ scrambled shock to the grid of the goalbox upon the interruption of the third photocell by the animal.

Procedure. Upon arrival in the laboratory, the subjects were individually housed and allowed free access to food and water for 10 days. The subjects were then placed on a 12-g daily fooddeprivation schedule, with continuous free access to water. In addition to their daily ration of $12 \mathrm{~g}$ of Purina Lab Chow, the animals received approximately 10 pellets of Purina Hog Starter per day for 10 days to familiarize them with the reward. During these 10 days, the animals were individually removed from their cages and handled for 2-3 min daily. Prior to the start of the first experimental manipulation, all subjects received two rewarded pretraining trials per day for 2 days. On rewarded (R) trials during pretraining and throughout the experiment, $2 \mathrm{~cm}$ of Purina Hog Starter (approximately $150 \mathrm{mg}$ ) was placed in the food cup. On a delayed (D) trial, the delay door prevented the animal's immediate access to the food cup for $30 \mathrm{sec}$. On a punished $(P)$ trial, the animal received the appropriate $.5 \mathrm{sec}$ of .2-mA shock immediately upon interrupting the third goalbox photocell and was allowed immediate access to the same magnitude of reward as on $R$ and $D$ trials. The combination of shock and reward was used on $P$ trials to avoid confounding the effects of punishment and nonreward. It should be noted that $.5 \mathrm{sec}$ of shock did not appreciably delay the eating of the pellets. Nonrewarded $(\mathrm{N})$ trials during both phases of the experiment consisted of a 30 -sec nonreinforcement confinement duration, during which time the empty food cup was accessible. The training phase lasted 12 days, and the testing phase was 6 days. All groups received four trials per day throughout the experiment; the intertrial interval (ITI) was approximately $5 \mathrm{~min}$. The animals spent the ITI in a neutral box with neither food nor water available.

During the first, acquisition phase, the three experimental groups received a $75 \%$ reinforcement schedule with three $R$ trials per day and one N, P, or D trial. Group N was a partial reinforcement group. It received three $R$ trials per day and one $\mathrm{N}$ trial (12 N-R transitions). Group PD received three $R$ trials per day and either one $P$ trial or one $D$ trial per day (six P-R and six D-R transitions). Group NPD received three $R$ trials per day and either one $\mathrm{N}$ trial, one $\mathrm{P}$ trial, or one $\mathrm{D}$ trial per day (four N-R transitions, four P-R transitions, and four D-R transitions). The aversive events occurred on the first, the second, or the third trial of the day. In addition, the three experimental groups were divided into three subgroups each $(n=3,3$, and 2$)$, so that the nature and/or the order of the aversive event received each day was counterbalanced within each group. For example, the 1st day of training for Group NPD consisted of the three schedules NRRR, PRRR, and DRRR, one for each of the three NPD subgroups. The CRF control group received four $R$ trials per day throughout the 12-day acquisition phase. During the second, testing phase, all groups received four $\mathrm{N}$ trials per day for 6 days.

\section{Results and Discussion}

There were no terminal acquisition differences among the four groups. One-way analyses of variance performed on the daily means of the last acquisition day did not reveal any significant differences $[\mathrm{Fs}(3,28)=.94$, $.95, .86$, and .95 for the start, run, goal, and total measures, respectively; ps $>.05$ ]. In terms of resistance to extinction, the groups were ordered N $>$ NPD $>$ PD $>\mathrm{CRF}$, providing support for the assumptions advanced earlier. Four 4 (groups) by 6 (days) repeated-measures analyses of variance performed on the daily means of all four measures revealed significant groups main effects $[F s(3,28)=8.92,11.05,11.32$, and 13.92 for 
the start, run, goal, and total speeds, respectively; ps $<.001]$, indicating significant differences among the four groups' resistance to extinction. The smallest days main effect was in the run section $[F(5,140)=45.50$, $\mathrm{p}<.001]$. In additionn, the Groups by Days interactions in the start, goal, and total speeds were significant $[\mathrm{Fs}(15,140)=2.30,2.16$, and 3.32 , respectively; ps $<.05$ ], but this interaction in the run speed measure was not significant $[F(15,140)=1.17, p>.25]$, indicating that the rate of extinction of the four groups differed in the start, goal, and total speeds measures, but not in the run speeds measure. A series of Tukey's pairwise comparisons conducted on the daily extinction means of all four groups in all four measures indicated that Group $\mathrm{N}$ was significantly $(\mathrm{p}<.05)$ more resistant to extinction than were groups PD and CRF and that Group NPD was significantly $(p<.05)$ more resistant to extinction than was Group CRF. Comparisons between adjacent groups did not reveal any significant differences. The latter finding should not detract from the overall importance of the data, especially the fact that the predicted ordering of the four groups was obtained in all four speed measures. Figure 1, showing the total speeds of the four groups during extinction, is representative of the extinction performance of the four groups in all four measures.

The results of this experiment provide support for the first two assumptions advanced here and indirect support for the summation assumption. It is clear that the specific transfer of habit strength (Group N) results in less generalization decrement than does nonspecific transfer (Group PD). It may also be inferred that habit strengths undergoing specific and nonspecific transfer summate (Group NPD) and yield stronger persistence than does habit strength undergoing nonspecific transfer alone (Group PD).

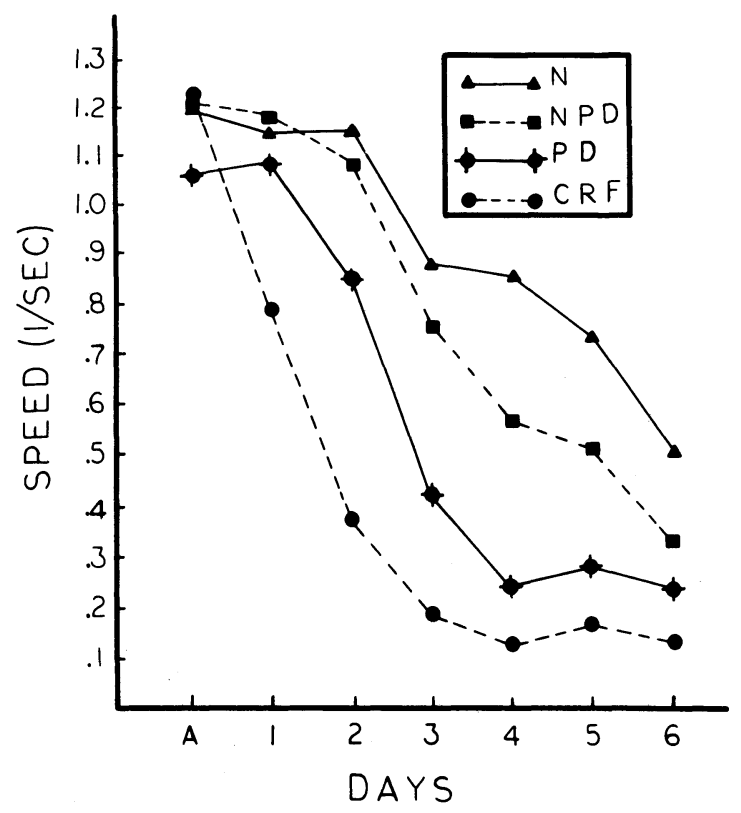

Figure 1. Mean total speeds for the last day of training (A) and the 6 days of extinction in Experiment 1.

\section{EXPERIMENT 2}

An adequately strong test of the summation assumption can be made simply by comparing the persistence of a group that receives only specific transfer with that of a group that receives the same specific transfer training as the first group, as well as other aversive events that would result in additional nonspecific transfer. Thus, a group that receives six N-R, six P-R, and six D-R transitions (Group XR) should be more resistant to extinction than a group that receives six $\mathrm{N}-\mathrm{R}$ transitions only (Group NR). Since the number of $N$ trials and the number of N-R transitions is held constant, the superiority of the first group can be accounted for only on the basis of the summation assumption.

In addition, a third group was included to test the relevance of sequential variables to the transfer of persistence. This third group (Group $\mathrm{RX}$ ) received six $\mathrm{N}-\mathrm{R}$ transitions and six $\mathrm{D}$ and six $\mathrm{P}$ trials, but the $\mathrm{D}$ and the $P$ trials were given on the last trial of the day, allowing for a D-R or a P-R transition on 24-h ITIs only. The number and percentage of $P$ and $D$ trials were, therefore, the same as those received by Group XR. Since $P$ and $D$ trials are placed at the end of the daily trial sequence for Group RX, only the six N-R transitions should influence persistence if sequential variables are indeed responsible for the development of persistence. Thus, Groups RX and NR should yield the same level of persistence, which should be inferior to that of Group XR. If, on the other hand, Groups XR and RX show equivalent levels of persistence that are superior to Group NR, one would have to conclude that persistence is controlled by an intratrial mechanism such as Amsel's (1972) mechanism, rather than by Capaldi's (1967) sequential variables.

\section{Method}

Subjects. The subjects were 27 rats similar to those used in Experiment 1 in all respects except age: They were 150 days old. The animals were randomly assigned to one of three groups ( $\mathrm{n}=9$ /group).

Apparatus. The apparatus was the same as that used in Experiment 1.

Procedure. Upon arrival in the laboratory, the animals were housed, handled, prefed, deprived, and pretrained like those in Experiment 1 . The ITIs and the parameters for $R, N$, and D trials were the same as those used in Experiment 1. P trials, however, consisted of $.5 \mathrm{sec}$ of $.1-\mathrm{mA}$ scrambled shock in addition to the appropriate amount of food pellets.

The training phase consisted of 18 days ( 54 trials), and the testing phase consisted of $3 \mathrm{~N}$ trials per day for 6 days. During acquisition, the number of trials per day was varied. All groups received two, three, or four trials per day. This procedure was used to insure the absence of patterning (anticipation of the last trial) in Group RX, which received the P and D trials on the last trial of the appropriate day. Thus, Group RX received $R$ trials following each of its six $\mathrm{N}$ trials (specific transfer), but it did not receive any trials (except $24 \mathrm{~h}$ later) following any of its $P$ or D trials (no nonspecific transfer). The six N, six P, and six D trials of Group XR, however, were each followed by at least one $R$ trial, resulting in specific transfer due to the six $\mathrm{N}-\mathrm{R}$ transitions and nonspecific transfer due to the six P-R and six D-R transitions. Finally, the six N trials received by Group NR were each followed by at least one $R$ trial, resulting in specific 
transfer only. The order of the number of trials per day over days was counterbalanced such that 18 different orders were used, in which any one number of daily trials (two, three, or four) was never repeated on more than 2 consecutive days.

\section{Results and Discussion}

There were no terminal acquisition differences among the three groups. One-way analyses of variance performed on the means of the last three acquisition trials did not reveal any significant differences $\left[F_{s}(2,24)=\right.$ $1.30, .83, .52$, and .14 for the start, run, goal, and total measures, respectively; ps $>.25$ ].

In terms of resistance to extinction, the groups were ordered $\mathrm{XR}>\mathrm{RX}=\mathrm{NR}$. Four 3 (groups) by 6 (days) repeated-measures analyses of variance performed on the daily means of all four measures revealed significant groups main effects in the goal measure $[\mathrm{F}(2,24)=$ $8.42, p<.01]$ and in the total measure $[F(2,24)=$ $4.93, \mathrm{p}<.05]$, but not in the start or the run measures. It should be noted here that the predicted orderings of the three groups was evident in all four measures, although the differences in the start and run measures were not significant. Figure 2, which shows the total speeds of the three groups during extinction, illustrates clearly the superior resistance to extinction of Group XR relative to Groups $\mathrm{RX}$ and NR. This observation was confirmed by a series of Tukey's pairwise comparisons conducted on the daily extinction means of the three groups in the goal and the total measures. Comparisons between the daily means of Groups RX and NR did not reveal any significant differences. Group $X R$, on the other hand, was found to be significantly $(p<.05)$ more resistant to extinction than were Groups RX and NR on the last 4 days of extinction in the goal and the total speed measures.

This experiment provides support for the summation assumption, in that Group XR showed higher resistance to extinction than did Group NR. This finding is also relevant to the results of Experiment 1. It supports the conclusion that the number of $\mathrm{N}$ trials and the number of N-R transitions were not the sole variables controlling the level of persistence of the three experimental groups in Experiment 1. Rather, the summation of nonspecific transfer effects (Group PD in Experiment 1) and the summation of specific and nonspecific transfer effects (Group NPD in Experiment 1) must have played an important role in the observed persistence of the experimental groups in Experiment 1. Furthermore, the lack of differences between Group NR and Group RX in this second experiment illustrates clearly that the sequential ordering of aversive events and immediate reward rather than the number of such events is a crucial variable in the development of persistence.

\section{REFERENCES}

Amsel, A. Behavioral habituation, counter-conditioning, and a general theory of persistence. In A. H. Black \& W. F. Prokasy (Eds.), Classical conditioning II: Current research and theory. New York: Appleton-Century-Crofts, 1972.

Banks, R. K. Persistence to continuous punishment following

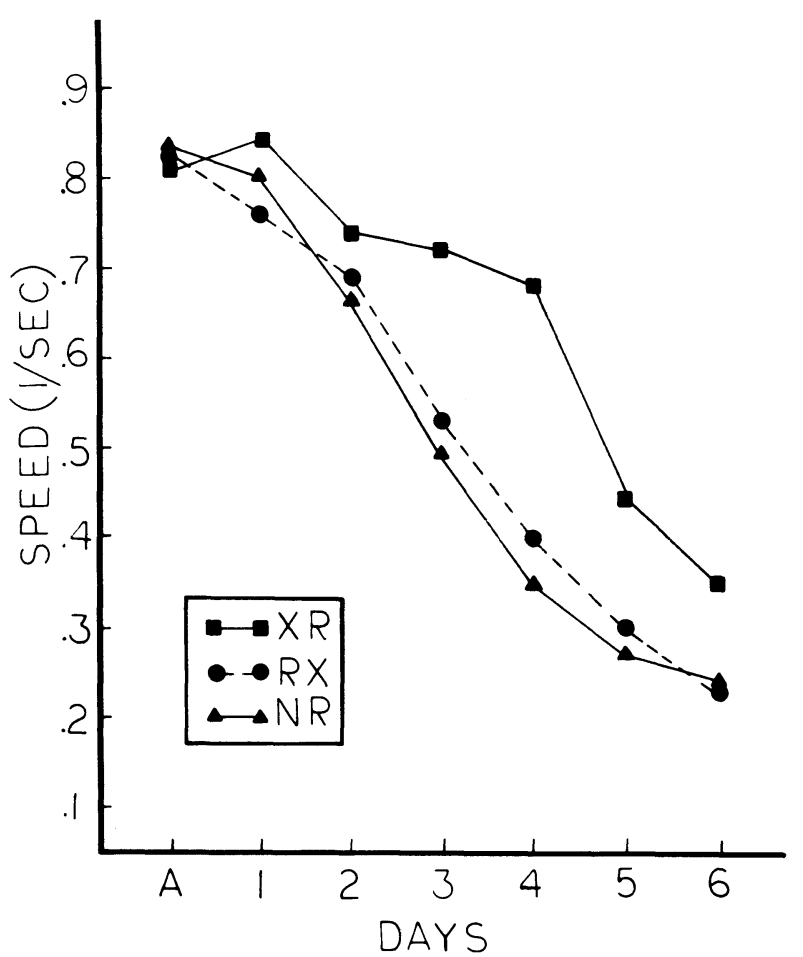

Figure 2. Mean total speeds for the last day of training (A) and the 6 days of extinction in Experiment 2.

intermittent punishment training. Journal of Experimental Psychology, 1966, 71, 373-377. (a)

Banks, R. K. Persistence to continuous punishment and nonreward following training with intermittent punishment and nonreward. Psychonomic Science, 1966, 5, 105-106. (b)

Banks, R. K., \& ToRney, D. Generalization of persistence: The transfer of approach behavior to differing aversive stimuli. Canadian Journal of Psychology, 1969, 23, 268-273.

Brown, R. T., \& W AGNER, A. R. Resistance to punishment and extinction following training with shock or nonreinforcement. Journal of Experimental Psychology, 1964, 68, 503-507.

CAPAldi, E. J. A sequential hypothesis of instrumental learning. In K. W. Spence \& J. T. Spence (Eds.), The psychology of learning and motivation (Vol. 1). New York: Academic Press, 1967.

Capaldi, E. J. An analysis of the role of reward and reward magnitude in instrumental learning. In J. Reynierse (Ed.), Current issues in animal learning. Lincoln: University of Nebraska Press, 1970.

Capaldi, E. J., \& Levy, K. J. Stimulus control of punished reactions: Sequence of punishment trials and magnitude of reinforcement trials. Learning and Motivation, 1972, 3, 1-19.

D'Aмато, M. R. Instrumental conditioning with negative reinforcement. In M. H. Marx (Ed.), Learning: Processes. New York: Macmillan, 1969.

Dyck, D. G., Mellgren, R. L., \& Nation, J. R. Punishment of appetitively reinforced instrumental behavior: Factors affecting response persistence. Journal of Experimental Psychology, $1974,102,125-132$.

Mellgren, R. L., Haddad, N., Williams, J. D., \& Conkright, R. K. Resistance to continuous delay of reinforcement or extinction following partial delay or partial reinforcement in acquisition: A direct comparison. Learning and Motivation, 1975, 6, 459-467.

Wagner, A. R. Frustrative nonreward: A variety of punishment. In B. A. Campbell \& R. M. Church (Eds.), Punishment and aversive behavior. New York: Appleton-Century-Crofts, 1969.

(Received for publication July 8, 1980.) 